extrêmeș et le beurrier devra veiller à réchauffer très légèrement sa crème en hiver, et à la rafraîchir judicieusement en été.

Un point, relativement méconnu, et sur lequel HunzIKER a ppelle l'attention des industriels beurriers, est celui de la mauvaise conductibilité des crèmes. Nous venons de voir qu'il faut, selon les circonstances, réchauffer légèrement ou rafraîchir énergiquement les crèmes. Or cette opération ne doit pas se faire au dernier moment parce que la crème est mauvaise conductrice de la chaleur. La modification de température doit être faite au moins deux heures a vant le barattage sous peine de ne pas atteindre le degré désiré.

Lorsque, par surprise ou par manque de temps, le beurrier est obligé de modifier la température de sa crème immédiatement a vant le barattage, il doit alors exagérer un peu son action dans un sens ou dans l'autre pour obtenir le résultát final cherché.

(A suivre).

\title{
LA MÉTHODE SYNTHÉTIQUE DANS L'ÉTUDE DU LAIT. LE LAIT AU POINT DE VUE COLLOIDAL. RECHERCHES SUR LE MÉCANISME DE L'ACTION DE LA PRÉSURE
}

\author{
par Ch. PORCHER, \\ Dooteur ès sciences physiques
}

(Suite)

\section{L'ACTION DE LA PRÉSURE SUR LES CASÉINATES}

Lorsque nous avons fait l'historique de l'emprésurage, nous avons dit, sans en donner en détail les raisons, que nous avions été conduit à accepter la théorie de Hammarsten, théorie chimique déjà ancienne, qui avait subi une éclipse presque totale. Nos premières vues nous en avaient éloigné nous-même et il n'a fallu rien moins qu'une dissection plus poussée du phénomène pour nous y ramener. Nous avons maintenant à montrer par quelle voie nous avons été conduit à adopter finalement la théorie de HAMMARSTEN sur le dédoublement de la caséine en paracaséine et en albumose du sérum.

\section{LA DISSEGTION DU PROGESSUS LABIQUE}

Puisque nous voulons aller du composé au simple pour revenir ensuite du simple au composé dans nos essais de synthèse des laits les plus variés, il est indiqué de séparer le caséinate de calcium des éléments salins qui lui sont associés dans le complexe et de faire porter l'action de. la présure uniquement sur le caséinate lui-même.

$\mathrm{Si}$, pour préparer le complexe phosphatique, nous partons d'un 
caséinate neutre, nous savons que nous devons lui ajouter de la chaux qui sera ensuite salifiée par l'acide phosphorique. Dans la préparation ordinaire du complexe phosphatique, nous nous adressons tout de suite à un caséinate calcique alcalin à 1100 , dans lequel 500 de chaux environ sont ultérieurement salifiés par l'acide phosphorique. Si nous partions du caséinate à 600 , nous aurions donc à lui ajouter 500 de chaux avant $d^{\prime} y$ apporter l'acide phosphorique.

Cette dernière façon de faire va nous guider dans notre dissection du processus labique :

Nous allons partir d'un caséinate d̀ 600 de $p \mathrm{H}=7$ environ, et le soumettre à l'action de la présure, puis, quand l'action labique sera épuisée, et par analogie avec ce que nous avons vu pour le complexe, nous y apporterons 500 de chaux et nous salifierons celle-ci par de l'acide phosphorique ajouté dans les mêmes proportions que s'il s'agissait du complexe luimême que nous préparerions en vue de l'emprésurer. Bref, les masses chimiques en présence seront les mêmes dans les deux cas, mais tandis que dans le premier, la présure agissait sur le complexe en son entier, dans le second, nous faisons d'abord porter son action sur le caséinate et ce n'est qu'après l'intervention fermentaire que nous apportons les compléments de chaux et d'acide phosphorique pour en venir chimiquement aux mêmes proportions que celles du complexe ordinaire. Ces considérations nous ouvrent tout un champ d'expériences que nous allons maintenant rappeler.

Quand nous faisons agir la présure sur le lait, il est impossible de saisir le moment où s'arrête la protéolyse labique et où commence la précipitation du paracaséinate ealcique. L'emprésurage, en effet, est un phénomène progressif à vitesses variables. Il est des cas où cette vitesse est considérable, et il en est d'autres, au contraire, où la coagulation est lente. Mais l'attaque de la caséine, puisqu'elle demande du temps, ne se fait pas instantanément et, entre le moment où commence l'action diastasique et celui où elle est tout à fait terminée, il est des stades intermédiaires où nous avons en présence de la caséine attaquée et de la caséine encore intacte, les proportions de cette dernière diminuant au fur et à mesure que s'accentue le phénomène.

On peut penser, puisque le caséinate est plus stable que le paracaséinate, que celui-là protège celui-ci jusqu'à un certain point : le moment où la quantité de paracaséinate sera telle qu'elle précipitera malgré la présence du caséinate non encore attaqué.

\section{LA PROTECTION DU PARACASÉINATE CALCIQUE PAR LE CASEINATE CALCIQUE.}

FuLd (267) a été le premier à envisager cette question de la protection du paracaséinate par le caséinate. Comme beaucoup de ses devanciers, il s'est employé à donner une théorie de la coagulation par 
la présure, mais à le lire, on sent qu'il hésite à fixer sa pensée. Séduit d'abord par les théories physiques, puisqu'il regarde le processus de la coagulation comme "un cas spécial des suspensions mutuelles et des précipitations des substances colloïdales ", après s'être prononcé, avec réserve toutefois, contre la théorie du clivage de HAMMARsTen, il semble par la suite y revenir, en faisant remarquer que la présure intervient en dehors de toute coagulation visible pour séparer de la easéine une partie de son azote ayant une valeur constante. Il termine son travail en faisant remarquer que lorsque la molécule de caséine est pure, elle est toujours attaquée par la présure, même en l'absence d'acide phosphorique, ou d'un autre acide, et si nous trouvons une albumose dans le sérum, cela tranche la question, dit-il, en faveur de la théorie de Hammarsten.

Lorsqu'il nous parle de considérer la coagulation comme un «cas spécial des suspensions mutuelles et des précipitations des substances colloïdales", c'est qu'il vise surtout le second stade du phénomène de la coagulation. Il pense que le paracaséinate de calcium n'est pas précipité par les sels calciques tant qu'une proportion suffisante ne s'en est pas formée pour surmonter l'influence stabilisante du caséinate. Lorsque celle-ci ne peut plus s'exercer, le paracaséinate entraînerait avec lui du caséinate non encore transformé. En d'autres termes, si nous avons bien saisi la pensée de FuLD, le caséinate de calcium ne protègerait le paracaséinate que dans une certaine limite qu'il nous semble facile de déterminer.

Pour ce faire, nous avons opéré de la façon suivante :

Nous prenons dù caséinate de calcium à 560 et nous le partageons en deux parties : l'une sera soumise à l'action de la présure (A), l'autre sera additionnée de la même quantité de présure, mais préalablement tuée (T).

Il est indispensable de tuer la présure dans le paracaséinate une fois que le ferment a cessé d'agir, parce que dans les mélanges en proportions variables de paracaséinate et de caséinate, nous aurions à craindre l'attaque ultérieure du caséinate par la présure venant du paracaséinate.

Nous avons done en présence, caséinate, d'une part, paracaséinate, de l'autre. Nous en faisons des mélanges en proportions variables. Ces mélanges sont stables comme les liqueurs de l'association desquelles ils sont obtenus.

Nous ajoutons à ces mélanges le $\mathrm{CaCl}^{2}$ pour y précipiter le paracaséinate.

Dans la solution de paracaséinate pur, l'addition du $\mathrm{CaCl}^{2}$ entraîne la formation immédiate d'un précipité : le caillé que l'on connaît, mais sous une forme floconneuse.

Dans les solutions où le paracaséinate domine, le précipité de paracaséinate de calcium déterminé par l'apport chloruro-calcique, entraînera peut-être avec lui le caséinate de calcium, partieliement ou en entier, mais a priori, on peut supposer que dans les solutions riches, au contraire, en caséinate, le $\mathrm{CaCl}^{2}$ ne produit pas de précipité; c'est que maintenant le caséinate de calcium sert de protecteur au paracaséinate. 
Nous avons procédé aux mélanges suivants :

$\begin{array}{rcc} & \text { TABLEAU CVI } \\ \text { Paracaséinate } & \text { Caséinate } \\ \text { I } & 10 & 0 \\ \text { II } & 9 & 1 \\ \text { III } & 8 & 2 \\ \text { IV } & 6 & 4 \\ \text { V } & 4 & 6 \\ \text { VI } & 2 & 8 \\ \text { VII } & 0 & 10\end{array}$

On les porte à $40^{\circ}$, et pendant qu'ils sont à cette dernière température, pour $10 \mathrm{~cm}^{3}$ de mélange, on ajoute $\mathrm{X}$ gouttes à la microburette de $\mathrm{CaCl}^{2} \mathrm{~N} / 2$ ce qui correspond à $1,11 \mathrm{gr}$. de $\mathrm{CaCl}^{2}$ par litre.

Ces gouttes sont versées à l'entrée du tube à essais qu'on a soin de tenir presque horizontalement ; ensuite, très brusquement, on renverse le tube de façon à mélanger rapidement les gouttes de $\mathrm{CaCl}^{2}$ à la liqueur.

Liqueur I. - Le précipité est tellement plastique que les flocons se contractent, se ramassent en une masse spongieuse. La synérèse est rapide, le sérum s'exsude clair et la masse floconneuse reste accolée à la paroi intérieure du tube à essais. Iei, il n'y a pas de caséinate, mais uniquement du paracaséinate.

Mélange II. - Déjà nous apercevons l'influence du caséinate. Le précipité se forme bien, mais le liquide qui exsude n'est plus limpide, il est trouble; il contient du caséinate. La caséine en précipite par acidification.

Mélange III. - L'action protectrice du caséinate est encore plus marquée. Le précipité au lieu d'être floconneux paraît granuleux. Il se rassemble en formant un petit culot au fond du tube, mais le liquide qui surnage est tout à fait trouble.

Melange IV. - Il en est ici comme des deux précédents avec une tendance encore plus accentuée.

Mélange $\nabla$. - Il y a au fond du tube un précipité un peu granuleux, moins volumineux que dans les cas précédents et qui se tasse aisément.

Melange $\nabla I$. - Très faible précipité au fond.

Liqueur VII. - Rien évidemment, puisqu'il ne s'agit que de caséinate.

Nous voyons qu'à partir du mélange de six parties de caséinate et de quatre de paracaséinate, l'action protectrice du caséinate est nettement marquée, au point que la précipitation du paracaséinate sous l'apport chloruro-calcique est pour ainsi dire entravée, ou du moins, n'est que très minime. Nous pouvions penser.qu'en forçant la dose de $\mathrm{CaCl}^{2}$, on parviendrait à la provoquer et surtout, à l'accentuer; or, nous avons doublé la dose de $\mathrm{CaCl}^{2}$ sans obtenir une précipitation plus marquée.

Mais il ne semble pas, comme le veut FuLD et comme on pouvait a priori l'admettre, que le paracaséinate entraîne du caséinate, puisque les précipités de colloïdes se chargent facilement d'autres colloïdes les entourant. Or, lorsqu'il y a un mélange de 9 parties de paracaséinate et de 1 de caséinate, la précipitation du paracaséinate laisse la liqueur trouble, un peu laiteuse, ce qui est dû au caséinate resté stable en solution. 


\section{L'APPRÉCIATION DU IMOMENT EXACT OU LE LAIT COAGULE. -}

L'expérience ci-dessus appelle des réflexions intéressantes concernant l'appréciation du moment où le lait coagule.

Si l'emprésurage, avons-nous dit, est un phénomène continu, il est cependant entendu, depuis qu'il a fait l'objet de recherches attentives par tous ceux qui l'ont étudié, que le moment où apparaît la floculation, puis la prise en masse qui l'accompagne, caractérise la fin du phénomène. Nul, jusqu'ici, ne s'est demandé pour quelle raison profonde, on choisissait ce moment. Evidemment, il est très caractéristique du point de vue objectif, mais quelle en est exactement la signification ? Voilà une questión qui doit être posée et à laquelle on doit s'efforcer de répondre.

A partir du moment où l'on met la présure dans le lait, l'attaque de l'édifice caséinique commence, elle se poursuit continument jusqu'au moment où la coagulation se déclanche, et le mot est assez juste étant donné parfois la rapidité de ce temps du phénomène. Pourquoi, en somme, y a-t-il floculation? L'étude de l'emprésurage sur le lait nous permet difficilement de donner une réponse précise et, une fois encore, c'est l'action de la présure sur un édifice caséinique, synthétiquement obtenu, qui permettra de satisfaire à notre question. Le paracaséinate de calcium est très fortement protégé par le caséinate calcique, non encore attaqué par la présure, et ce n'est que lorsque tout le caséinate a subi l'attaque de la présure que cette protection cesse-tout à fait, d'où, comme conséquence, la floculation qui n'est qu'une précipitation de paracaséinate calcique, sans caséinate parasite, et à laquelle fait suite immédiatement la soudure, c'est-à-dire la prise en masse, le bloc du caillé. Cette coagulation traduit donc l'attaque entière du caséinate et elle la justifie.

Les caséinates calciques seuls ne coagulent pas par la présure. - La présure réserve son action lytique au caséinate de calcium; mais alors qu'avec le complexe, il y a coagulation, ici, il n'en est plus de même, parce que la présure ne coagule pas les caséinates dont le $p H \leqslant 7$, quelle que soit leur charge calcique. Mais si elle ne les coagule pas, du moins provoque-t-elle leur dédoublement qui, objectivement, n'est pas accompagné d'un phénomène aussi marqué qu'une précipitation. Nous noterons seulement que te caséinate calcique emprésuré blanchit fortement. C'est que le paracaséinate calcique qui s'est formé est en imminence de précipitation. Il est indispensable que les caséinates calciques soient d'un $p \mathrm{H} \leqslant 7$, ou du moins que leur $p H$ ne dépasse pas $7,4-7,5$. Il faut également que la présure soit dialysée, qu'elle soit débarrassée de ses sels, notamment de tout le chlorure de sodium qu'elle contient en abondance, afin que nous puissions juger des phénomènes qui se passent sans être gêné par l'intervention de ce qué nous pouvons appeler l'impureté saline de la présure, impúreté évidemment nécessaire pour la préparation industrielle de eelle-ci, 
mais qui nous embarrasse dans nos expériences. Nous allons en instruire à nouveau la preuve. Il était utile de préparer des caséinates d'une grande concentration, plus de 30 grs par litre, parce qu'avec eux, il est toujours possible d'en obtenir des complexes au taux ordinaire par dilution ultérieure avec de l'eau de chaux, puis neutralisation de celle-ci par des acides variés.

L'ACTION DE LA PRÉSURE SUR LES CASEINATES EN SOLUTIONS ConcentreEs. - LE ROLE du NaCi de la PRÉsure. - Au début de nos recherches, en faisant agir de la présure sur des caséinates de $p H \leq 7$, renfermant de 60 à 100 grammes de caséine au litre, nous avions constaté une coagulation. Nous la mettions sur le compte de la forte concentration de la protéine, de l'accumulation de ses micelles qui venaient à se souder dès que la présure avait diminué un peu la viscosité du milieu. Mais la suite de nos expériences nous a permis d'incriminer la charge saline considérable de la présure, laquelle se monte on le sait, pour les présures liquides commerciales, à 250 grammes de $\mathrm{NaCl}$ au litre environ.

Dans ces conditions, quand, à un easéinate très concentré, nous apportons de la présure, elle-mêmo extrêmement riche en $\mathrm{NaCl}$, nous provoquons, par double décomposition, la formation d'une masse assez importante de $\mathrm{CaCl}^{2}$ et nous réalisons un système : caséinate de calcium + chlorure de calcium qui présipite, régulièrement, comme nous le savons, sous l'action du ferment-lab.

Nous avons préparé un caséinate à 600 au taux de 60 grammes de caséine par litre nécessitant done 1200 de chaux (caséinate double, comme nous l'appellerons), puis un caséinate à 400 à 100 grammes de caséine par litre, contenant done 3,33 fois plus de caséine que notre caséinate habituel, et en chaux, 1333 au litre.

$$
\begin{aligned}
& p \text { H } \\
& \text { Caséinate à } 600 \text { (60 grs). . . . . . . . 7,07 } \\
& \text { Caséinate à } 400 \text { (100 grs). . . . . 6, 6, 65 }
\end{aligned}
$$

Nous avons opóré parallèlement avec de la présure liquide non dialysée et de la présure dialysée. A $2 \mathrm{~cm}^{3}$ de caséinate, nous ajoutons $1 / 20$ de $\mathrm{cm}^{3}$ de présure.

Avec le caséinate à 60 grammos ot à 100 grammes, la coagulation est rapide et ne demande que quelques 'secondes quand nous opérons avec de la présure non dialysée; la synérèse s'exerce vite et laisse exsuder un sérum tout à fait limpide.

Si nous nous servons, au contraire, de présure dialysée, nous n'obtenons pas de coagulation, dans un cas comme dans l'autre; la liqueur blanchit, devient tout à fait porcelanique, mais ne présente pas la moindre floculation.

Les ealculs nous permettent de voir que, au taux de 250 grammes de sel par litre, la quantité de présure non dialysée 'apporte, selon la quantité employée, 6,25 grammes de $\mathrm{NaCl}$ par litre de caséinate.

\section{DIMINUTION DE LA VISCOSITÉ DES CASÉINATES PAR EMPRÉSURAGE.}

Quand on regarde les choses de pròs, on peut constater que le caséinatè 
modifié par la présure est moins visqueux que le caséinate originel. La couche qu'il forme sur la paroi du tube à essais s'amincit plus rapidement et s'écoule plus vite.

On prépare des caséinates doubles à $400,500,600$ et 650 de chaux contenant 60 grammes de caséine au litre. Par conséquent, il nous faudra doubler la quantité de chaux et prendre $800,1000,1200$ et 1300 de chaux.

Voici quels sont les $p H$ de ces caséinates immédiatement après leur préparation et après 24 heures :

\begin{tabular}{|c|c|c|c|c|c|c|c|c|}
\hline & & & & \multirow[t]{2}{*}{ TABLEAU } & \multirow{2}{*}{\multicolumn{2}{|c|}{ CVII }} & \multicolumn{2}{|c|}{$p$ H } \\
\hline & & & & & & & $\begin{array}{l}\text { Immédiatement } \\
\text { apress }\end{array}$ & $\begin{array}{c}\text { Au bout } \\
\text { de } 24 \text { heures }\end{array}$ \\
\hline Caséinate & à 400 . & . & . & $\ldots$. & . . & . & 6.38 & 6.20 \\
\hline » & 500. & & &... & . . & & 6.58 & 6.37 \\
\hline 》 & 600 . & . & & $\therefore .$. & $\cdots$ & & . $\quad 7.15$ & 7.05 \\
\hline 》 & 650. & . & & . . . . & . . & & 7.45 & 7.38 \\
\hline
\end{tabular}

Nous avons ici un nouvel exemple de ce que nous avons appelé la maturation des caséinates.

Trente-six heures après leur préparation, ces caséinates sont emprésurés à $40^{\circ}$ pendant 15 minutes. A $25 \mathrm{~cm}^{3}$ de chacun d'eux, on ajoute $3 \mathrm{~cm}^{3}$ de présure dialysée (A).

Pour le témoin, la présure a été au préalable chauffée au B-M, bouillant pendant 5 minutes de façon à la tuer $(\mathrm{T})$.

Voici les temps d'écoulement notés au viscosimètre Baume :

TABLEAU CVIII. Présure

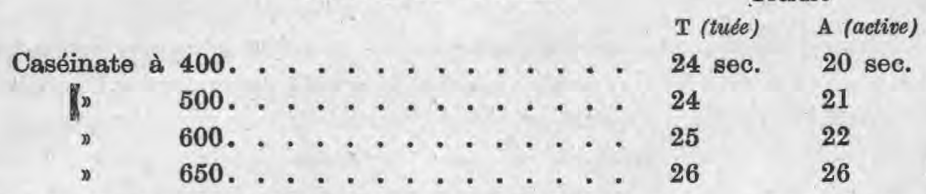

Notons que les caséinates à 400 et à 500 sont acides, celui à 600 , neutre ( $p$ H voisin de 7 ), celui de 650 alcalin (son $p H=7,6$ ).

Les chiffres de ce tableau sont très intéressants; ils nous montrent que l'action protéolytique du lab s'exerce d'autant mieux que le caséinate est plus acide. Avec le caséinate à 650, elle ne s'exerce plus ; il n'y a pas de différence de viscosité entre les deux caséinates $T$ et $A$, ce qui est la preuve que nulle action digérante n'a été exercée par la présure dans ce cas.

Une autre expérience nous a donné les résultats suivants :

TABLEAU CIX. Temps d'écoulement

$T$ A

Caséinate à 600 préparé depuis 3 jours . $\quad 28$ sec. $\quad 23$ sec.
Caséinate à 550 venant d'être préparé. . - $\quad 36$ sec. $\quad 24$ sec.
Caséinate à 650 venant d'être préparé. . . 41 sec. $\quad 41$ sec.

Comme précédemment, on note que lorsque le caséinate est alcalin, au point de ne pouvoir être attaqué par la présure, il n'y a pas de différence entre $T$ et $\mathbf{A}$. 
On doit également remarquer que lorsque le caséinate vient d'être préparé, il est plus visqueux. Nous relevons, en effet, dans le tableau cidessus que le caséinate à 550 , fraîchement préparé, demande au viscomètre de Baume 36 secondes pour s'écouler entre les deux repères de l'appareil; celui de 650, 41 secondes, alors que dans l'expérience qui précède, - il s'agissait de caséinates préparés depuis 24 heures -, les temps d'écoulement sont nettement moincres : 25 secondes pour le caséinate à 600,24 pour le caséinate à 550 .

\section{COMMENT SE RENDRE COMPTE CHIMIQUEMENT DE L'ATTAQUE} du caséinate PAR LA PRÉsure? - Tout simplement, comme nous l'avons fait pressentir plus haut, en chargeant ce caséinate emprésuré de phosphates de calcium dans les mêmes conditions où nous opérons avec le caséinate témoin qui a reçu la même quantité de présure préalablement tuée par la chaleur.

Nous préparons un caséinate double à 600 (60 gr. de caséine, 1,2\% de chaux). Il est en effet nécessaire qu'il soit double, puisque après l'action de la présure, nous avons à l'additionner de chaux, puis d'acide phosphorique, ce qui en amène la dilution, ear nous nous arrangerons toujours pour avoir finalement un caséinate à 30 grammes, comme dans le complexe.

On en prend deux portions de $50 \mathrm{~cm}^{3}$, l'une (T) est additionnée de $1 \mathrm{~cm}^{3}$ d'une présure dialysée, préalablement bouillie, l'autre (A) est additionnée de la même quantité de la même présure, mais non bouillie.

Les deux liqueurs sont chauffées au B-M à $40^{\circ}$. On laisse 15 minutes à cette température, beaucoup plus de temps qu'il n'en faut pour amener, avec la quantité de présure mise en cuvre, la coagulation du complexe que nous préparerions dans les conditions habituelles où nous opérons.

Nous avons donc en présence les deux caséinates $\mathrm{T}$ et $\mathrm{A}$; apparemment, ils ne paraissent pas très différents; le caséinate A est un peu plus blanc que le $\mathrm{T}$. Il n'y a pas de précipitation dans A.

C'est de ces deux liqueurs dont nous allons partir maintenant pour faire le complexe. Mais il est indiqué de tuer la présure ajoutée dans l'échantillon A. P our cela, on le porte à $70^{\circ}$ pendant un quart d'heure et pour que la comparaison sur les deux échantillons $\mathbf{A}$ et $\mathbf{T}$ soit aussi parfaite que possible, nous chauffons également l'échantillon $\mathrm{T}$ dans les mêmes conditions.

Le chauffage à $70^{\circ}$ n'amène aucun trouble dans $T$, alors que dans $A$, nous relevons autour de la ouvette du thermomètre qui nous servait à agiter la liqueur pour empêcher la formation possible d'une "peau ", une précipitation un peu gluante, visqueuse, mais en somme très faible, qui n'est autre que du paracaséinate calcique. Si toute la paracaséine ne précipite pas, c'est que la quantité de chaux en présence est insuffisante. Ce chauffage à $70^{\circ} \mathrm{n}$ 'est d'ailleurs pas indispensable ainsi que nous l'avons reconnu par la suite.

Comme nous partons toujours de easéinates à 1100 pour faire les complexes, il était indiqué d'ajouter 500 de chaux à notre caséinate à 600 . Les liqueurs qui étaient blanchâtres sont redevenues translucides, puis nous avons apporté l'acide phosphorique.

Les liqueurs T' et A qui à l'origine étaient de $50 \mathrm{~cm}^{2}$ ont été ramenées à $45 \mathrm{~cm}^{3}$ 
par les chauffages à $40^{\circ}$ et $70^{\circ}$ auxquels elles ont été soumises. On complète dans les deux cas a $50 \mathrm{~cm}^{3}$, puis on ajoute encore $6,5 \mathrm{~cm}^{3}$ d'eau distillée, $40 \mathrm{~cm}^{3}$ d'eau le chaux de façon à faire un caséinate à 1100 en chaux, et $3,5 \mathrm{~cm}^{3}$ d'acide phos. phorique, ce dernier acide étant versé goutte à goutte de la burette micrométrique, sous l'agitation électrique, et on arrive ainsi à $100 \mathrm{~cm}^{3}$.

Quand on charge le témoin T et qu'on a fini de faire écouler l'acide phosphorique, on obtient un complexe laiteux, très homogène, qui, additionné de présure à $40^{\circ}$ coagule normalement en donnant un bloc solide, ferme. Tout cela est régulier; la présure qu'on avait donc ajoutée en premier lieu était réellement tuée, et nous voyons une fois de plus qu'un caséinate de $p H \leqslant 7$ peut subir l'action de la température à $70^{\circ}$ dans les conditions ei-dessus relevées sans être altéré.

Mais c'est avec l'échentillon A que nous allons obtenir quelque chose de particulièrement intéressant : alors que nous sommes sur le point d'arriver à la fin de l'addition phosphorique, une précipitation rapide du complexe se fait en grumeaux irrégulièrement soudés laissant une liqueur un peu opalescente.

C'est là une belle expérience que nous avons répétée et en voici le détail :

\section{Caséine : 15 grammes}

Eau de chaux (1,4 gr. par litre) : $214 \mathrm{~cm}^{3}$

Quand toutes les opérations seront terminées, les $15 \mathrm{gr}$, de caséine devront être dilués à $500 \mathrm{~cm}^{3}$ de façon à bien avoir 30 grammes au litre. Le caséinate ainsi préparé était à 600 de chaux, et son $p$ H $=7,07$.

Préparation de la liqueur $T: 21,5 \mathrm{~cm}^{3}$ dudit caséinate +5 d'eau ; $2,5 \mathrm{~cm}^{2}$ de présure dialysée et préalablement chauffée à $100^{\circ}$.

Préparation de la liqueur $A$ : Mêmes quantités, mais la présure n'est pas chauffée.

Les deux caséinates sont chauffés une demi-heure à $40^{\circ}$.

On a porté ensuite à $70^{\circ}$ pendant 20 minutes, ce qui amène la mort du ferment dans $A$ et l'on agite pendant qu'on chauffe. Le caséinate $T$ ne présente rien de particulier, tandis que le caséinate $A$ a un aspect un peu différent : de fines particules de paracaséinate sont adhérentes aux parois du ballon, mais dans son ensemble, la liqueur est restée homogène et très opaque. Les deux ballons sont refroidis sous un courant d'eau. On ajoute à chacun d'eux $18,5 \mathrm{~cm}^{3}$, d'eau de chaux et $2 \mathrm{~cm}^{3} \mathrm{~d}^{\prime} a c i d e$ phosphorique.

Dans le cas du tube $T$, on obtient un complexe d'un espect ordinaire comme ceux que nous préparons en partant du caséinate à 1100 , son $p H=6,84$. Il coagule par la présure dialysée $\left(5 \mathrm{~cm}^{3} \mathrm{du}\right.$ complexe $+0,25 \mathrm{~cm}^{3}$ de présure $)$ en donnant un caillé ferme au bout de 30 secondes.

Avec le tube A, au contraire, avant d'en arriver à la fin de la dose nécessaire de $2 \mathrm{~cm}^{3}$ d'acide phosphorique, alors qu'on en est à $1,5 \mathrm{~cm}^{3}$, de petits coagulats se forment, et en continuant l'addition phosphorique jusqu'à $2 \mathrm{~cm}^{3}$ comme pour le tube $T$, on obtient finalement et très rapidement une masse qui se ramasse en un bloc autour de l'agitateur en laissant un liquide clair.

Dans ces expériences, nous voyons que sur le caséinate emprésuré la présure ayant été tuée après qu'elle a agi - l'addition du complément de chaux étant réalisée, la précipitation du complexe par l'acide phosphorique a lieu avant que tout l'acide ne soit ajouté. Nous avons 
noté le phénomène massif, e'est-à-dire, la précipitation. Il y a lieu maintenant d'examiner de très près les conditions dans lesquelles elle s'exerce. C'est pourquoi nous avons fait varier les temps d'emprésurage du caséinate afin de voir si, lcomme conséquence de cette variation, la précipitation du complexe, lors de l'addition phosphorique, se ferait à des moments différents.

On prépare un caséinate double à 550 (1,83 \%) e'est-à-dire à 60 grammes de caséine et 1100 de chaux au litre.

A $25 \mathrm{~cm}^{3}$ de ce caséinate, on ajoute $2,5 \mathrm{~cm}^{3}$ de présure dialysée ; cette quantité eoagule le complexe ordinaire que nous connaissons bien en 15 secondes.

On fait 5 essais dans lesquels on fait varier les temps d'action de la présure, qui sont respectivement : $30 \mathrm{sec}$., 1 min., 5 min., 15 min., 30 min., à $40^{\circ}$, c'est-à-dire, que tous ces temps sont supérieurs à celui de 15 secondes qui est suflsant pour coaguler le complexe correspondant servant de témoin, ainsi que nous venons de le voir.

Après l'emprésurage de ces caséinates, on porte immédiatement chacun des tubes au B-M bouillant en les faisant tourner très rapidement de façon à renouveler les surfaces et atteindre vite la température de $70^{\circ}$ qu'on conserve pendant 15 minutes, puis on refroidit.

Un témoin a reçu la même quantité de la même présure, mais préalablement tuée par un chøuffage à $100^{\circ}$.

Avant de continuer l'expérience, on constate que les caséinates emprésurés (A) sont des liquides blancs, porcelaniques, tout à fait laiteux, alors que le caséinate témoin (T) est toujours quelque peu translucide.

A chacun des caséinates ci-dessus, emprésurage fait et présure tuée, on ajoute $18,50 \mathrm{~cm}^{3}$ d'eau de chaux à $1,4 \mathrm{gr}$. au litre pour obtenir finalement un caséinate à 1100 avec 30 grammes de caséine au litre, puis de l'eau distillée pour compléter à $48 \mathrm{~cm}^{3}$. Il reste $2 \mathrm{~cm}^{3}$ qui sont réservés pour l'addition phosphorique.

Nous ferons remarquer que lorsqu'on ajoute le complément de chaux aux caséinates avant de les charger en acide phosphorique, on aboutit dans tous les cas à des liqueurs d'une extrême translucidité, presque transparentes qui ne se distinguent pas l'une de l'autre, qu'il s'agisse de caséinate emprésuré ou non, et quand nous envisageons les caséinates emprésurés, que l'emprésurage ait duré 30 secondes ou 30 minutes.

Quand on ajoute l'acide au caséinate témoin (T), on n'observe rien d'anormal et on obtient un complexe blanc, légèrement opalescent.

Avec les caséinates emprésurés, on constate, lorsqu'on verse l'acide phosphorigue très lentement avec la microburette, qu'immédiatement après l'addition des premières gouttes, il y a formation de nuages filamenteux plastiques, difficiles à disloquer, visqueux, finissant au fur et à mesure qu'ils augmentent par se souder les uns aux autres en donnant des masses irrégulières ayant l'aspeet spongieux.

Si on constate de petites différences selon les flacons, elles tiennent sans aucun doute à des agitations plus ou moins régulières.

Dans le flacon correspondant à l'emprésurage de 30 minutes, la liqueur paraît très homogène pendant l'agitation ininterrompue, au point qu'il peut sembler ne pas s'être fait de précipité. L'addition phosphorique terminée, on arrête l'agitation, et on recueille autour de l'agitateur une masse tremblotante, constituée de la soudure de caillés très fins et très visqueux qui se rétractent petit à petit en laissant exsuder le sérum, tout eomme cela se voit avec un caillé de lait. 
Bref, nous n'avons pas obtenu de différences essentielles entre les liqueurs quels que soient les temps d'emprésurage qui vont de 30 secondes à 30 minutes. Au fond, cela ne doit pas nous surprendre, puisque déjà la coagulation du complexe témoin était réalisée au bout de 15 secondes. En d'autres termes, l'action protéolytique nécessaire à l'emprésurage est épuisée déjà au bout de 15 secondes, aussi bien dans le complexe que dans les caséinates.

Les sérums de tous les essais ci-dessus n'ont pas la limpidité de l'eau distillée, limpidité que possède le sérum d'emprésurage du témoin, puisque le caillé est agité pendant qu'il se forme, mais il filtre clair après plusieurs passages sur papier.

L'expérience ci-dessus nous conduit naturellement à voir ce que l'on obtiendrait si l'emprésurage du caséinate durait moins de temps qu'il n'en faut au complexe témoin pour coaguler par la présure. Il faut envisager l'expérience d'une autre façon et s'adresser à deux caséinates identiques, mais qu'on emprésure pendant des temps différents, l'un en deçà, l'autre, au delà du temps nécessaire pour que le complexe témoin fasse prise.

Une présure étant donnée, nous l'avons diluée pour apprécier sa force de façon que $25 \mathrm{~cm}^{3}$ du complexe témoin $+2,5 \mathrm{~cm}^{3}$ de présure coagulent en 5 minutes. $25 \mathrm{~cm}^{3}$ de caséinate double $+2,5 \mathrm{~cm}^{3}$ de cette présure sont laissés à $40^{\circ}$, un premier échantillon 2 minutes, l'autre 15 minutes, et, comme dans l'expórience antérieure, on tue la présure lorsque l'emprésurage est terminé. Notons qu'à ce moment, les deux caséinates sont différents. Celui dont l'emprésurage a duré 15 minutes est tout à fait blanc laiteux, porcelanique, alors que celui qui n'a été soumis que 2 minutes a l'action de la présure est encore un peu translucide avec une teinte légèrement jaune ambrée.

L'addition de la chaux complémentaire donne des liqueurs identiques : translucidité marquée, presque transparence

C'est la charge en aceide phosphorique qui va nous donner des résultats tout à fait dissemblables.

Avec le caséinate emprésuré pendant 2 minutes, on obtient tout d'abord, bien que l'écoulement de l'acide soit très lent, des petits flocons qui finissent par être dilacérés par l'agitateur, au point que lorsque tout l'acide est ajouté, la liqueur paraît à première vue homogène comme le témoin. Mais si l'on regarde de près, on voit, lorsqu'on penchele tube et qu'on le relève, que la mince couche de liquide qui s'écoule est semée de très fins précipités, qui grossissent si on porte à $40^{\circ}$ au B-M sans grande tendance toutefois à la soudure. Ils nagent au milieu d'un liquide encore très opaque, très blanchâtre. Si on chauffe à $60^{\circ}$, ils grossissent encore davantage.

Dans cette liqueur, manifestement, l'action de la présure n'a pas été complète. Si on l'emprésure à nouveau à froid et qu'on la porte à $40^{\circ}$, on a une parfaite coagulation avec une prise en masse, le deuxième emprésurage ayant porté sur la partie du complexe encore inaltéré.

Quant au tube dans lequel l'emprésurage a duré 15 minutes, les 
résultats que nous donne l'addition phosphorique sont identiques à ceux qu'on a enregistrés plus haut.

Dans toutes ces expériences, nous sommes fondé à dire que le précipité obtenu lors de l'apport phosphorique correspond à celui que nous donne l'emprésurage d'un complexe. Mais alors qu'avec le complexe, le sérum est limpide comme de l'eau distillée, ici, et nous l'avons déjà noté, le sérum obtenu est un peu trouble, ce qui est dû au battage mécanique des palettes de l'agitateur lors de l'arrivée de l'acide phosphorique. Mais en fait, lorsqu'on l'a filtré plusieurs fois sur papier, le filtrat est tout à fait limpide, tout au plus, a-t-il une très légère opalescence. Ce filtrat renferme de la chaux, de l'acide phosphorique et une protéine, car il donne toutes les réactions des protéines : précipité avec le réactif d'ESBACH, avec le réactif de TANRET, avec l'azotate mercurique. Il donne la'réaction de Mrtion.

Cette protéine a été engendrée par le processus d'emprésurage; elle répond à ce que HAMMARSTEN a appellé l'albumine du sérum (molkeneiweiss). C'est une protéose qui résulte du clivage de la caséine par la présure, et il va de soi que ce clivage doit toujours se faire de la même façon dès l'instant où les circonstances qui l'entourent sont également les mêmes.

Un nouveau plan de recherches se présente à nous de ce morceau détaché de la molécule de la caséine par la présure, et des données à la fois qualitatives et quantitatives doivent être obtenues.

Il importe, en effet, que nous sachions quel est le poids $d u$ morceau détaché de la molécule de caséine par la lyse labique. En d'autres termes, partant d'un poids donné de caséine, quelle sera l'importance pondérale de la protéine solubilisée par l'emprésurage.

ii est certain que la conception de HAMMARSTEN vers laquelle tendent nos recherches, puisque celles-ci admettent que la caséine est scindée en deux morceaux d'inégales grosseurs, est plus facile à justifier par le recours à la méthode synthétique. En opérant sur le complexe pur, nous ne sommes pas embarrassé par les autres protéines : albumine, globuline qu'on trouve dans le lait. Si donc, un dédoublement de la caséine s'opère libérant un peptide qui, soluble dans l'eau, se retrouvera dans le sérum, l'analyse nous permettra de le peser, d'y rechercher le phosphore, le soufre, etc.. d'y déterminer le quantum de son azote carbaminable.

Nous avons expérimentalement des moyens de nous rendre compte quantitativement d'abord, qualitativement ensuite, de ce que peut être ce morceau détaché de la molécule.

\section{LES DIVERSES MÉTHODES A EMPLOYER POUR LE CALCUL DE LA PROTÉOSE'SOLUBLE DETACHEE DE LA CASEINE PAR LA PRESURE.}

La détermination du poids de la protéose est d'une réelle importance 
pour la justification de l'hypothèse de Hammarsten à laquelle nous nous rallions. Si nous en établissons le bien fondé, en même temps que nous justifions l'hypothèse sus-dite, nous rejetons les autres, notamment celle de VAN SLYKe et Bosworth qui admet le dédoublement de la caséine en deux moreeaux dont la masse moléculaire serait la moitié de celle de la caséine; dans ce cas, il n'y aurait pas de protéose soluble formée lors du clivag.

Les difficultés rencontrées. - Avant de montrer de quelle façon nous sommes arrivé à mesurer assez exactement le poids de la protéose soluble de Hammarsten, il est nécessaire de se mettre en face des difficultés que l'on peut rencontrer et qui tiennent :

a) pour une part, d̀ ce que nous opérons sur des milieux colloïdaux dont l'aspect micellaire est toujours hétérogène, et,

b) pour une autre part, à ce que très facilement, le moindre excès d'acide aboutit à des acido-solubilisations, lesquelles influencent ainsi la masse propre de la protéose soluble. Du reste, nous reviendrons sur la grande attention qu'il faut apporter toutes les fois que dans la mesure du morceau détaché de la caséine par le clivage labique, nous a vons à faire intervenir les acides forts.

Plaçons-nous en face des cireonstances variées dans lesquelles l'emprésurage va s'effectuer et voyons comment elles vont se prêter à la mesure du morseau détaché par la présure.

Le recours au complexe phosphatique. - Lorsque nous faisons l'emprésurage sur le complexe : caséinate de calcium + phosphates de calcium, complexe qui nous a servi habituellement dans nos recherches, nous aboutissons par l'addition de présure dialysée, - et c'est évidemment toujours celle-ci dont il faut se servir en semblable circonstance, - à un caillé ferme dont la synérèse s'effectue énergiquement en laissant exsuder un sérum extrêmement clair.

Nous trouvons, d'une part:

a) dans le coagulum: le paracaséinate de calcium + des phosphates de calcium insolubles; d'autre part :

b) dans le sérum: la protéose soluble et les phosphates solubles de calcium.

Dans ces conditions, un premier obstacle se présente dès l'instant où les sels, les phosphates calciques solubles, se trouvent dans le sérum à côté de la protéose. Il nous est, en effet, difficile de savoir si dans la protéose soluble, il existe du phosphore lui appartenant en propre et qui antérieurement se trouvait dans la caséine. Pour éviter des erreurs d'interprétation et de calculs, il faut donc de toute nécessité ne pas s'adresser au complexe phosphatique ordinaire, puisque son emprésurage laisse passer dans le sérum les phosphates calciques solubles du complexe originel. 
LE Recours Au Complexe chloruro-calcique. - Lorsque nous voulons savoir si la présure détache du phosphore de la caséine, nous devons donc, de toute nécessité, nous adresser à un autre système qui ne renferme en phosphore que celui qui appartient en propre à la molécule protéique qui va être clivée. Nous y parviendrons en travaillant sur l'édifice : caséinate de calcium + chlorure de calcium. Si dans son attaque par la présure, nous trouvons dans le sérum du $\mathrm{PO}^{4} \mathrm{H}^{3}$, nous sommes dans l'obligation d'admettre que dans la partie solubilisée par le lab, il est du phosphore organique, en liaison, bien entendu, avec la masse azotée de la protéose.

Dans ces conditions, voyons, comme nous l'avons noté tout à l'heure avee le complexe phosphatique, ce qu'il y a dans le coagulum, d'une part, et dans le sérum d'autre part.

Le coagulum est-il constitué uniquement de paracaséinate calcique? C'est vraisemblable. Dans le sérum, nous trouvons toujours la protéose soluble et le chlorure calcique ajouté pour l'emprésurage.

le recours au complexe carbonatique. - Nous pouvons également mesurer la partie soluble détachée dans le processus labique, en ayant recours au système : caséinate de calcium + carbonates de calcium.

A près l'emprésurage, le coaǵulum très rétieulé que nous connaissons, n'est autre chose que du paracaséinate de calcium éminemmentplastique, et dans le sérum, nous trouvons, à côté de la protéose soluble, du bicarbonate calcique et en outre du carbonate neutre précipité si le liquide est laiteux, et du gaz carbonique qui se dégage en toutes petites bulles.

Avec les trois complexes ou systèmes salins, que nous venons d'utiliser : le phosphatique, le chloruro-calcique et le carbonatique, l'acidité du milieu était celle que nous désirions, et nous pouvions procéder à l'emprésurage tout à fait au voisinage de 7 .

Au lieu d'emprésurer les complexes une fois obtenus, nous poúvons faire porter l'action du ferment-lab sur les caséinates neutres de $p \mathbf{H}=7$ environ, puis procéder à l'addition ultérieure de la chaux et de l'acide.

En l'espèce, on opèrera de la façon suivante :

On prend un caséinate calcique double (caséine: $60 \mathrm{gr}$. et chaux 1,20 gr. par litre) de $p H \leqslant 7$, voisin de 7. On l'emprésure. Aucune précipitation, bien entendu, à la température ordinaire ; la liqueur en ce moment renferme un mélange de paracaséinate calcique et de protéose soluble. Sans qu'il soit alors nécessaire de tuer la présure qui s'y trouve et qui est encore active, — nous n'avons rien à craindre de son action, parce que nous allons opérer rapidement, - nous additionnons 500 de chaux à la liqueur faite avec un caséinate à 600 , ce qui a pour but de la rendre très alcaline, et nous revenons au $p \mathrm{H}$ voisin de 7 , dans un cas, par apport phosphorique, dans l'autre, par apport chlorhydrique, et dans le troisième, par apport carbonique. Nous savons que nous allons déterminer dans les trois cas, un précipité de paracaséinate calcique; 
ce présipité, avec le complexe phosphatique, sera chargé de phosphate trical sique, alors qu'avec le complexe chloruro-calcique il n'y aura pas de eharge ealcique insoluble. Dans le cas où c'est $\mathrm{CO}^{3} \mathrm{H}^{2}$ qui intervient, le paracaséinate précipitant pourra englober du carbonate neutre de calcium.

Si nous envisageons le sérum obtenu après neutralisation du paracaséinate calcique par $\mathrm{PO}^{4} \mathrm{H}^{3}, \mathrm{HCl}$ ou $\mathrm{CO}^{3} \mathrm{H}^{2}$, nous savons que nous trouverons toujours dans ce sérum la protéose soluble, mais dans le premier cas, elle sera accompagnée de phosphates calciques solubles, dans le second cas, de chlorure de calcium, et dans le troisième cas, de bicarbonate de calcium.

Le grand avantage de recourir au complexe : caséinate de calcium + carbonates de calcium, est que l'acidification par l'acide carbonique a une limite qu'on ne saurait dépasser. Son $p H$, nous l'avons vu, est de 6,40 , traduisant une acidité plus que largement suffisante pour aider à l'emprésurage.

Lorsque nous opérons avec le complexe chloruro-calcique, au lieu d'emprésurer le caséinate double, puis de l'additionner de chaux et d'acide chlorhydrique, on peut encore s'adresser au caséinate simple dans lequel, après l'action' complète du lab, on versera une quantité suffisante de $\mathrm{CaCl}^{2}$ pour provoquer la précipitation du paracaséinate de calcium.

Avec les méthodes ci-dessus, nous opérons toujours en présence de charges salines assez importantes, celle des phosphates dans le complexe phosphatique, celle des carbonates dans le complexe carbonatique, celle du chlorure de caleium dans le système chloruro-calcique. Nous allons maintenant travailler sur le caséinate sans addition d'une charge saline étrangère; après emprésurage du caséinate, on en précipitera la paracaséine.

La précipitation de la paracaséine laisse la protéose en solution. - Nous préparons un caséinate de calcium de $p H$ voisin de 7 . Faisons deux parts de la liqueur; l'une sera soumise àl'actiondela présure (A), l'autre sera considérée comme témoin (T), Retenons que dans toutes les recherches dont nous parlons en ce moment, dès l'instant où nous procèdons par comparaison, nous devons toujours déterminer le taux de l'azote qui sera apporté par la présure dans nos liqueurs, de façon à en tenir compte. Il est faible, c'est certain, mais il n'est pas négligeable.

Dans les deux portions de la solution de caséinate, nous aurons done à ajouter, dans l'une, de la présure tuée (T) par chauffage, et dans l'autre, de la présure active (A), vivante, si nous pouvons nous exprimer ainsi. Mais l'apport azoté, dans l'une comme dans l'autre, aura toujours été semblable.

Lorsque l'action de la présure est terminée, nous avons donc dans une liqueur, celle qui a reçu de la présure active $(\mathrm{A})$, du paracaséinate 
calcique et la protéose soluble, et dans l'autre, celle qui a reçu la présure tuée ( $T)$, le caséinate qui n'a pas été modifié.

Si donc, on apporte de l'acide dans les deux liqueurs pour atteindre le point isoélectrique : $p H=4,6-4,7$, nous précipiterons dans la liqueur qui a reçu de la présure tuée, la caséine, et dans celle qui a reçu dẹ la présure active, la paracaséine.

Si nos précipitations sont bien faites, si l'apport acide est rigoureusement exact. dans la première liqueur nous n'avons pas de protéose soluble, puisqu'il ne s'en est point fait ; l'azote soluble n'appartient qu'à la présure apportée. Au contraire, dans la seconde liqueur où nous avons précipité la paracaséine, l'azote soluble appartient, d'une part, à la présure apportée comme tout à l'heure, mais aussi à la protéose soluble de Hammarsten.

Si on opérait sans grande attention et que l'apport acide ne fut pas suivi goutte à goutte, - et encore doit-on se servir de liqueurs diluées, nous risquerions d'avoir des acido-solubilisations qui viendraient troubler profondément nos dosages d'azote.

Dans les méthodes dont nous venons de donner le principe pour le calcul du morceau détaché par la présure, nous avons toujours eu recours au caséinate calcique et nous avons mis en jeu l'insolubilisation du paracaséinate calcique en présence d'une charge minérale calcique soluble suffisante. Mais avec la dernière méthode qui aboutit à la précipitation de la paracaséine, au lieu de travailler sur un caséinate calcique, nous pouvons nous adresser à un caséinate alcalin: de sodium ou de potassium, en ayant soin, bien entendu, que son $p \mathrm{H}$ soit toujours dans la zone acide. Dans ces conditions, rien de changé quant au fond des opérations, nous précipitons caséine et paracaséine de leurs sels alcalins, comme nous l'avons fait tout à l'heure de leurs sels calciques, en ayant soin, comme toujours, de surveiller attentivement l'arrivée de l'acide.

Les diverses méthodes que nous venons d'exposer en vue de voir ce qu'elles peuvent donner pour la mesure de la protéose soluble sont à base chimique. Nous pouvons recourir à une autre qui est physique : l'ultrafiltration.

L'ULTRAFILTRATION POUR LA SEPARATION DE LA PROTÉOSE SOLUBLE. - Si l'ultrafiltration porte sur une liqueur au sein de laquelle se trouvent en solution paracaséinate et protéose soluble, e'est-à-dire sur un caséinate de $p \mathrm{H} \leq 7$ emprésuré, la protéose soluble traverse, car elle est filtrable, tandis que le paracaséinate est retenu.

En fait, les choses sont plus compliquées, parce que l'ultrafiltration d'une partie soluble en présence d'une protéine insoluble jouissant, comme la paracaséine, d'une grande plasticité, est rendue difficile par des phénomènes de colmatage, d'adsorption.

Nous verrons plus loin ce que l'emploi des méthodes dont nous venons 
d'exposer le principe donne dans la mesure du morceau détaché de la caséine par la lyse labique.

\section{LA PRÉPARATION DE LA PARACASÉINE.}

Pour le moment, nous allons donner un mode de préparation nouveau de la paracaséine qui dérive tout naturellement de nos recherches et qui va nous permettre l'obtention de cette protéine dans des conditions très précises.

Les auteurs qui ont voulu préparer de la paracaséine en s'adressant au lait lui-même, ont rencontré beaucoup de difficultés pour aboutir à un produit pur.

A.W. Bosworth et H.I. Bowditch (288) donnent le'mode de préparation ci-dessous qui va nous permettre, en effet, de justifier ce que nous venons de dire.

Le lait écrémé est coagulé par l'addition de présure, et quand le caillé est devenu suffisamment ferme pour que le doigt, quand on l'y enfonce, donne une section nette, il est divisé en petits morceaux; on en enlève le sérum et on le lave plusieurs fois avec de l'eau. Si le caillé est obtenu d'une laiterie, il doit être brisé et on le broie à travers un moulin à viande.

Le caillé finement divisé est maintenant placé dans un large récipient propre et on lui ajoute einq volumes d'eau pour chaque volume de lait employé pour l'obtenir, puis on le díssout en ajoutant environ $10 \mathrm{~cm}^{3}$ d'eau ammoniacale concentrée pour chaque litre de lait employé. Comme le caillé se dissout lentement, on agite le temps qui est nécessaire pour obtenir sa dissolution.

Lorsque la solution complète est obtenue, la paracaséine est précipitée de la façon suivante : on prend $2,5 \mathrm{~cm}^{3}$ d'acide acétique glacial pour chaque litre de lait employé et on le dilue avec 25 volumes d'ear. On ajoute cet acide dilué à la solution de paracaséine, très lentement, en agitant d'une façon constante. L'acide précipite rapidement la paracaséine en gros flocons qui vont au fond du vase et une attention soutenue doit être apportée à cette opération parce qu'un excès d'acide la redissoudrait. Si un précipité très fin apparaît, lequel ne se dépose pas rapidement, c'est qu'il s'est formé un sel calcique insoluble de paracaséine, et l'addition d'une plus grande quantité d'acide en le décalcifiant libérera la protéine. Une quantité d'acide plus ou moins grande que celle qui a été spécifiée ci-dessus peut alors donner une précipitation eonvenable. Tout cela dépend des conditions de l'âge du lait et du caillé, et de la quantité d'ammoniaque utilisée pour dissoudre ce dernier. Après que la paracaséine a déposé, on enlève le liquide surnageant, puis on la lave plusieurs fois avec de l'eau et on la redissout en employant le même volume d'eau que plus haut, en prenant la précaution de ne pas ajouter un excès d'ammoniaque, car cela causerait une hydrolyse de la paracaséine avec une perte du rendement. Le mieux est de diluer l'ammoniaque avee 50 volumes d'eau et d'employer tout juste ce qu'il en faut pour dissoudre la paracaséine. Après complète redissolution, celle-ci est de nouveau précipitée avec l'acide acétique dilué, et lavée comme auparavant.

(288) A.W. Bosworth et H.I. Bowditch, - Casein in infant feeding. The Preparation of Dry Powdered Paracasein and Preliminary Experiments Concerning its Use as the Principal Protein Constituent in Infant Food. The Amer Journ. of Diseases of Children, 1914, 8, 120-126. 
La technique à employer dépend du point de savoir si l'on veut obtenir un produit tout à fait pur. A chaque précipitation, il se forme en effet, du paracaséinate de calcium insoluble, qui est entraîné avec une masse beaucoup plus forte de paracaséine libre, et e'est seulement par des précipitations répétées que tout le calcium est enlevé. Afin d'assurer l'obtention d'une paracaséine libre de cendres, il est nécessaire de faire six ou sept précipitations. (53) Avee deux précipitations, on obtient un produit assez bon.

Après la précipitation finale et le lavage, la paracaséine est mise dans un sac de toile pour qu'elle s'égoutte pendant deux heures, puis on la porte dans un mortier et on la triture avec de l'alcool à $95 \%$. Ensuite, elle se rassemble au fond, on décante l'alcool et on triture à nouveau avec de l'alcool frais en répétant l'opération deux ou trois fois. La déshydratation est complétée en laissant la paracaséine en contact avee un grand volume d'alcool à $95 \%$ pendant une heure ou deux. Cet alcool est enlevé par décantation, on filtre sur un linge de toile et, si on le veut, on porte le tout dans une presse pour enlever l'excès d'alcool. La préparation est ensuite pulvérisée, et enfin séchée entre 80 et $90^{\circ}$.

Avec tous les soins nécessaires, le produit final est une poudre fine. Si la consistance de poudre n'est pas obtenue, on fait passer dans un moulin à café. Le produit final contient aveo deux précipitations : $1,7 \%$ d'humidité, $0,4 \%$ de cendres et $98 \%$ de paracaséine. Il est insoluble dans l'eau, légèrement soluble dans une solution à $5 \%$ de chlorure de sodium, complètement et facilement soluble dans une solution diluée de carbonate de sodium. La substance préparée de cette manière est de la paracaséine et non pas de la caséine.

Nous savons que pour L.L. van Styke et A.W. Bosworth (289), une molécule de caséine est dédoublée par la présure en deux molécules de paracaséine. Ces auteurs estiment que l'action de la présure s'exerce sur la caséine d'une façon tout à fait semblable à celle qui conduit au dédoublement du maltose en deux molécules de dextrose.

Nous avons voulu donner en détail la technique de A.W. BoswortH et H.I. BowDirTch pour montrer combien elle est entourée de difficultés : la pénible solubilisation du caillé d'emprésurage dans l'ammoniaque, les grandes précautions qu'il faut prendre pour éviter un excès d'acide lorsqu'on prézipite la paracaséine, pour éviter un excès d'ammoniaque lorsqu'on la redissout, nous montrent qu'il faudrait que précipitations et redissolutions fussent suivies exactement au potentiomètre. En admettant même que cela fût fait, la préparation de la paracaséine en partant d'un milieu aussi complexe que le lait nécessite des redissolutions et des reprécipitations successives assez nombreuses.

Notre technique. - Au contraire, si l'on a recours à la technique que nous préconisons : préparation d'un caséinate calcique ou sodique de $p H<7$, emprésurage, puis précipitation au point isoélectrique de la paracaséine par un acide très dilué apporté à froid avec précaution sous une forte agitation, nous facilitons singulièrement les opérations, nous

(289) A. W. Boswortr, - The action of Rennin on Cssein, Journal of biol. Chem. 1913, 15, 231 . 
les réduisons en même temps que nous opérons en présence d'une charge minérale la plus réduite possible. Deux précipitations et redissolutions successives seront suffisantes pour l'obtention d'une paracaseine vraiment pure. Nous pourrons opérer ici, comme nous l'avons fait pour la préparation de la caséine : lavages à l'alcool, à l'rither, etc...

(A suive).

\section{BIBLIOGRAPHIE ANALYTIQUE}

\section{LES LIVRES}

A. Dervaux. - L'élevage des animaux domestiques en Annam. Thèse Doct. Vét., Paris. Une brochure de 50 pages, imprimerie Watrelot, Armentières, 1929.

L'auteur nous donne quelques aperçus géographiques sur l'Annam, puis nous parle de l'élevage et des différents animaux domestiques en Annam. Retenons ce qu'il dit des bovidés et des buffles.

Il termine par quelques considérations sur les épizooties.

Ch. P.

\section{A. Brenot, - L'industrie laitière dans le département du Jura.}

Thèse Doct, Vét., Lyon, 1929. Une brochure de 66 pages avec deux cartes, Lyon, Imprimerie Bose et Riou, 1929.

Dans ce travail, B. nous fait l'histoire des fruitières du Jura et de leur fonctionnement, histoire précédée d'ailleurs d'une étude du milieu au point de vue de la production laitière. Ce travail est fort bien rédigé et il est accompagné de deux cartes très claires. Dans l'une, le Jura se trouve divisé en ses principales régions, et dans l'autre, nous trouvons la liste complète des fromageries et des beurreries coopératives. C'est done un document utile à consulter pour ceux que l'industrie laitière du Jura peut intéresser, let ils doivent être nombreux, étant donnée l'importance de cette industrie de ce département.

Ch. $\mathrm{P}$.

Leçons du jeudi soir à la clinique Tarnier. - $5^{\mathrm{e}}$ année, 1928. Un volume de 225 pages a vec 71 figures. Vigot frères, ćd., Paris, 1929.

Tous les ans, sous la direction du Professeur A. Brindeau, sont organisées, le jeudi soir, en hiver, des leçons sur des sujets d'obstétrique ou voisinant avec eux. Dans le volume de cette année, nous signalerons la leçon faite par le Professeur Ch. Porcher : "Pourquoi le lait de femme ne coagule-t-il pas par la présure ? " Faisant appel à la méthode synthétique qui lui a tant servi dans son livre: Le lait au point de vue colioĩdal, Ch. Porcher donne les raisons pour lesquelles le lait de femme ne coagule pas par la présure. Illles résident dans la présence d'une grande quantité de globuline et dans une variation de la structure saline du lait.

Au surplus, dans Le Lait paraîtra en 1931 une étude plus complète sur le lait de femme.

A. Girard,

\section{Carl Neubera. - Travaux de I'Institut Empereur-Guillaume de Berlin-Dahlem pour la biochimie, 1928.}

Ce volume rassemble une série de travaux faits dans le laboratoire de notre 